\title{
Nanobridged rhombic antennas supporting both dipolar and high-order plasmonic modes with spatially superimposed hotspots in the mid-infrared
}

\author{
En-Ming You ${ }^{1, \dagger}$, Yiqin Chen ${ }^{2, \dagger}$, Jun Yi ${ }^{1, \dagger}$, Zhao-Dong Meng ${ }^{1}$, Qian Chen ${ }^{1}$, \\ Song-Yuan Ding ${ }^{1 *}$, Huigao Duan ${ }^{2 *}$, Martin Moskovits ${ }^{3}$ and \\ Zhong-Qun Tian ${ }^{1}$
}

\begin{abstract}
Mid-infrared antennas (MIRAs) support highly-efficient optical resonance in the infrared, enabling multiple applications, such as surface-enhanced infrared absorption (SEIRA) spectroscopy and ultrasensitive mid-infrared detection. However, most MIRAs such as dipolar-antenna structures support only narrow-band dipolar-mode resonances while high-order modes are usually too weak to be observed, severely limiting other useful applications that broadband resonances make possible. In this study, we report a multiscale nanobridged rhombic antenna (NBRA) that supports two dominant resonances in the MIR, including a charge-transfer plasmon (CTP) band and a bridged dipolar plasmon (BDP) band which looks like a quadruple resonance. These assignments are evidenced by scattering-type scanning near-field optical microscopy (s-SNOM) imaging and electromagnetic simulations. The high-order mode only occurs with nanometer-sized bridge (nanobridge) linked to the one end of the rhombic arm which mainly acts as the inductance and the resistance by the circuit analysis. Moreover, the main hotspots associated with the two resonant bands are spatially superimposed, enabling boosting up the local field for both bands by multiscale coupling. With large field enhancements, multiband detection with high sensitivity to a monolayer of molecules is achieved when using SEIRA. Our work provides a new strategy possible to activate high-order modes for designing multiband MIRAs with both nanobridges and nanogaps for such MIR applications as multiband SEIRAs, IR detectors, and beam-shaping of quantum cascade lasers in the future.
\end{abstract}

Keywords: optical antenna; charge transfer plasmon; multiband resonances; scanning near-field optical microscopy; surface-enhanced infrared spectroscopy

You EM, Chen YQ, Yi J, Meng ZD, Chen Q et al. Nanobridged rhombic antennas supporting both dipolar and high-order plasmonic modes with spatially superimposed hotspots in the mid-infrared. Opto-Electron Adv 4, 210076 (2021).

\footnotetext{
${ }^{1}$ State Key Laboratory of Physical Chemistry of Solid Surfaces, Collaborative Innovation Center of Chemistry for Energy Materials, College of Chemistry and Chemical Engineering, Xiamen University, Xiamen 361005, China; ${ }^{2}$ National Engineering Research Center for High Efficiency Grinding, College of Mechanical and Vehicle Engineering, Hunan University, Changsha 410082, China; ${ }^{3}$ Department of Chemistry and Biochemistry, University of California, Santa Barbara, California, 93106, USA.

tThese authors contributed equally to this work.

"Correspondence: SY Ding, E-mail: syding@xmu.edu.cn; HG Duan, E-mail: duanhg@hnu.edu.cn

Received: 17 June 2021; Accepted: 3 September 2021; Published online: 30 December 2021
}

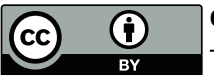

Open Access This article is licensed under a Creative Commons Attribution 4.0 International License.

To view a copy of this license, visit http://creativecommons.org/licenses/by/4.0/.

(C) The Author(s) 2021. Published by Institute of Optics and Electronics, Chinese Academy of Sciences. 


\section{Introduction}

Mid-infrared antennas (MIRAs), often constructed from metals (e.g., $\mathrm{Au}, \mathrm{Al}$ or $\mathrm{Ag}$ ), highly doped III-V semiconductors, electron-doped graphene or phonon-polaritonbased nanostructures ${ }^{1-11}$, support optical resonance in the mid-infrared spectral range (400 to $4000 \mathrm{~cm}^{-1}$ ). MIRAs can act as receiving antennas thereby concentrating mid-infrared beams from free space to nanoscale regions (termed as hotspots) in the vicinity of the surface of MIRAs ${ }^{12}$. MIRAs can also act as transmitting antennas to directionally amplifying thermal radiation produced by local heating of sources coupled to MIRAs ${ }^{13}$. These impressive features of MIRAs have inspired a wide range of investigation of their potential applications for surface-enhanced infrared absorption (SEIRA) spectroscopy leading to ultrahigh sensitivities (up to hundreds of oscillators $)^{14}$, for biological and chemical sensors in the mid-infrared region ${ }^{15-18}$, for beam-shape engineering of quantum cascade lasers $(\mathrm{QCL})^{19}$, and for highly-responsive photodetector $\mathrm{s}^{20}$ with enhanced absorption and photocarrier collection efficiency in the mid-infrared ${ }^{21}$. The core elements for the high-performance applications are the MIRA micro- and nanostructures, but the development of MIRA structures lags far behind that of optical antenna nanostructures in the visible spectral range $\mathrm{e}^{22}$.

Single-arm dipolar-antenna structures are among the most classical MIRAs, often consisting of gold rods with tunable resonant wavelengths by tuning the length of the $\operatorname{rods}^{23-26}$. Furthermore, dual-arm dipolar-antennas with nanometer-sized gaps (nanogaps), such as gold rod dimers, have also been developed on account of the strength of the local field enhancement factors (LFEFs, $\left.\left|\boldsymbol{E}_{\mathrm{loc}} / \boldsymbol{E}_{0}\right|^{2}\right)$ in their nanogaps ${ }^{27,28-29}$. Nevertheless, both single-arm and dual-arm dipolar-antennas usually support only the dipolar resonance mode which is a fundamental and narrow-band mode with a typical bandwidth around $200-500 \mathrm{~cm}^{-1}$. Usually, high-order modes in single-arm or dual-arm are typically too weak in the optical spectra ${ }^{26,28,30}$. This feature limits the application demanding multiple resonances in MIR region, such as SEIRA with a broad range so as to measure molecular vibrational absorption bands in fingerprint range $500-1500 \mathrm{~cm}^{-1}$ and functional group range $1500-$ $4000 \mathrm{~cm}^{-131}$.

To obtain multiband MIRAs, several micro- and nanostructures beyond single-arm or dual-arm antennas have been designed, among them, gold nano-crosses ${ }^{32,33}$, nanoaperture structures ${ }^{34,35}$, fractal microstructures ${ }^{36-38}$, log-periodic trapezoidal structures ${ }^{39}$, and dipolar antennas with multiple length ${ }^{40-42}$. These structures could be categorized into the micro- and nanostructures supporting several dipolar modes. Fundamentally, it is a longterm challenge to develop single-arm or dual-arm antennas supporting simultaneously pronounced fundamental and high-order plasmonic modes such as a quadrupolar mode.

In this study, we develop a nanobridged rhombic antenna (NBRA) exhibiting two pronounced resonancebands in the MIR regions. The two bands are assigned to a charge-transfer plasmon (CTP) mode and a bridged dipolar plasmon (BDP) mode, which are demonstrated by the scattering-type scanning near-field optical microscopy (s-SNOM), a technique that is widely used to image the near-field distribution of plasmonic modes ${ }^{43-47}$. The nanobridge structure and the linked rhombic-arm antennas effectively controls the resonant frequencies and extinction intensities of the CTP and BDP bands. The s-SNOM also demonstrates that the main hotspots of the two bands are spatially overlapped. This feature enables us to boost up the local field enhancement of both bands via NBRA dimers with nanogaps. Moreover, one order of magnitude of additional enhancement can be obtained for both bands from a hybrid structure consisting of an NBRA dimer, a sandwiched dielectric spacer layer and a gold reflector. Such high field enhancement enables detecting a monolayer of molecules using the two absorptions located in the two bands.

\section{Materials and methods}

\section{Numerical simulations}

All electromagnetic simulations were performed by the commercial software COMSOL Multiphysics based on the finite element method. The simulation space was a cuboid, whose sizes along the long and short axes of the NBRA were equal to the fabricated arrays. The perfectly matched layers were used for the top and bottom boundaries while the periodic conditions were employed for the side boundaries. The polarization of the incident electric field was either parallel or perpendicular to the long axis of the antenna and the resulting transmittance or reflectance spectra were averaged to approximate the unpolarized illumination used in the experiment. Corners and edges of the gold nanostructure were rounded by a radius of $10 \mathrm{~nm}$ for a reasonable simulated local-field distribution there. The mesh size was set as 0.5 $\mathrm{nm}$ for each dimension of the nanostructure and its close proximity and gradually became coarser toward the outer 
borders of the simulation domain. The mesh sizes of different parts are listed in Table S1. The convergence condition is satisfied (Fig. S2, Supplementary information). All the $\left|\boldsymbol{E}_{\mathrm{z}}\right|$ and $\varphi_{\mathrm{z}}$ were evaluated in the plane $50 \mathrm{~nm}$ above the top surface of the NBRA structure. The LFEF was evaluated at the point $2 \mathrm{~nm}$ away from the extremity of the structure and $15 \mathrm{~nm}$ above the $\mathrm{CaF}_{2}$ substrate. The refractive index for $\mathrm{CaF}_{2}$ was 1.41, and the dielectric constants for gold were adapted from literature ${ }^{48}$.

\section{Fabrication of NBRAs}

All the NBRAs were fabricated on the $\mathrm{CaF}_{2}$ substrate or on the reflective substrate by sequentially using electronbeam lithography, metal deposition and "Sketch and Peel" lithography (SPL) ${ }^{49-53}$. First, a hydrogen silsequioxane (HSQ) resist (XR-1541-006, Dow Corning) was spin-coated on the substrate at the speed of $4000 \mathrm{rpm}$. Then, a conductive polymer (ESPACER, Showa Denko) layer of $30 \mathrm{~nm}$ was casted on the HSQ resist to avoid pattern distortions due to the charging effect. The sample was then directly loaded into electron-beam direct writing system (Raith 150 Two). The exposure was executed by an electron beam with an accelerating voltage of 30 $\mathrm{kV}$ and a beam current of $280 \mathrm{pA}$. Before the development, the top layer of conductive polymer should be removed by DI water for $10 \mathrm{~s}$, and then a salty developer (1\%wt $\mathrm{NaOH}+4 \%$ wt $\mathrm{NaCl}$ aqueous solution) was used to chemically etch the unexposed HSQ resist for $1 \mathrm{~min}$. To stop further development, the structured substrate was immediately rinsed by DI water. The dipping in isopropanol reduced the surface tension and further helped us to obtain the upright HSQ thin-wall structures on the substrate after blow-drying of nitrogen gas steam.

Metal deposition was applied by a thermal evaporation system. The working chamber was pre-pumped to the pressure of $1 \times 10^{-5} \mathrm{~Pa}$, and the working pressure was kept at the value of $5 \times 10^{-5} \mathrm{~Pa}$. Due to the requirement of peeling off gold film, the deposition of adhesive metal was excluded from our fabrication process. A 30-nm thick gold film was obtained at a rate of 5 angstrom/s. The thickness was monitored by a quartz-crystal microbalance possessing the sensitivity of angstrom.

The standard process of SPL was reported in our previous literature ${ }^{49-53}$. To figure out the influence of HSQ in optical measurement, a vapor-HF chemical etching was used to remove HSQ templates. To avoid the condensation of $\mathrm{H}_{2} \mathrm{O}$ byproduct in etching, the sample was kept at the temperature of $40^{\circ} \mathrm{C}$.

\section{Scanning electron microscopy}

The morphology of resultant substrates was characterized by a field-emission scanning electron microscope (SIMG-HD, Carl-ZEISS). To avoid the charging effect during the imaging process, the accelerating voltage and the working distance were set as $1 \mathrm{kV}$ and $3 \mathrm{~mm}$, respectively.

\section{FTIR microscopy measurements}

A commercial FTIR spectrometer (Thermo Fisher Nicolet iN10) was used to perform all transmittance and reflectance measurements. The instrument is equipped with a silicon carbide (globar) light source, a $\mathrm{KBr}$ beam splitter, Cassegrain objective $(15 \times$, N.A. $=0.4$, incident angle $\theta$ ranging between 10 and $44^{\circ}$ ) and a mercury cadmium-telluride (MCT) detector. The transmittance or reflectance of the antennas were defined as the signal intensities transmitted/reflected from the antenna divided by those from a background taken at a blank area on the substrate. Each spectrum was acquired by averaging 64 spectra with a $3 \mathrm{~cm}^{-1}$ spectral resolution. A $100 \times 100$ $\mu \mathrm{m}^{2}$ square collection aperture was used for all the measurements. The SEIRA spectra were obtained by subtracting the baseline (generated by asymmetric least-squares smoothing algorithm $)^{54}$ from the original data. The incident and collected light are unpolarized light.

\section{Scattering-type scanning near-field optical microscopy (s-SNOM) measurement}

The s-SNOM system (neaspec $\mathrm{GmbH}$ ) was employed to perform the near-field amplitude and phase imaging of the NBRA structures. A silicon atomic force microscopy (AFM) tip (Nanosensors, PPP-NCH) worked as the scatterer to transform the near-field signals to the far field, when the tip and the antenna sample were illuminated by the focused beam of a continuously tunable QCL source. The illuminating beam was incident at $\sim 50^{\circ}$ referring to the surface normal, and its polarization was parallel to the long axis of the NBRA structure (s-polarized light). The signals backscattered by the tip were detected with a pseudo-heterodyne interferometer ${ }^{55}$. The vertical ( $p$-polarized) components of the signals were selected by using a polarizer in front of the detector. To obtain a modulation of the distance between tip and antenna, the tip was oscillating vertically at a frequency $\Omega \approx 250 \mathrm{kHz}$ with an amplitude of $\sim 100 \mathrm{~nm}$. Demodulation of the detected signals at the third harmonic frequency $(3 \Omega)$ yielded almost background-free amplitude and phase signals $\left|s_{3}\right|$ and $\varphi_{3}$. 


\section{Functionalization with PNTP molecules of the} antennas

The sample was incubated in an ethanolic solution of 4nitrothiophenol (PNTP, 98\%, Matrix Scientific) for 12 hours and then was rinsed with a large amount of ethanol (AR, Sinopharm Chemical Reagent Co., Ltd) to remove physically adsorbed molecules, and finally was dried with nitrogen. The concentration of the ethanolic solution of PNTP was kept constant at $1 \mathrm{mM}$.

\section{Results and discussion}

\section{NBRA structure and optical properties}

The NBRA (Fig. 1(a, c) and Fig. S3(a), Supplementary in- formation) consists of two rhombic arms connected with each other by a nanobridge (about $30 \mathrm{~nm}$ in width and $130 \mathrm{~nm}$ in length). Each arm contains a sharp tip (the corner angle $\alpha$ in Fig. 1(d) is $30^{\circ}$ ) with a radius of curvature about $10 \mathrm{~nm}$. The thickness $\left(t_{0}\right)$ and total length $\left(l_{0}\right)$ of NBRA are $30 \mathrm{~nm}$ and $2800 \mathrm{~nm}$, respectively. The NBRA arrays were fabricated by the SPL method which is appropriate for fabricating multiscale metallic patterns with high-fidelity features and nanogaps (see Materials and methods for details $)^{49-53}$. The periodicities along the long and short axis of the NBRA are $3600 \mathrm{~nm}$ and $1000 \mathrm{~nm}$. The experimental and simulated transmittance spectrum (Fig. S4, Supplementary information), as
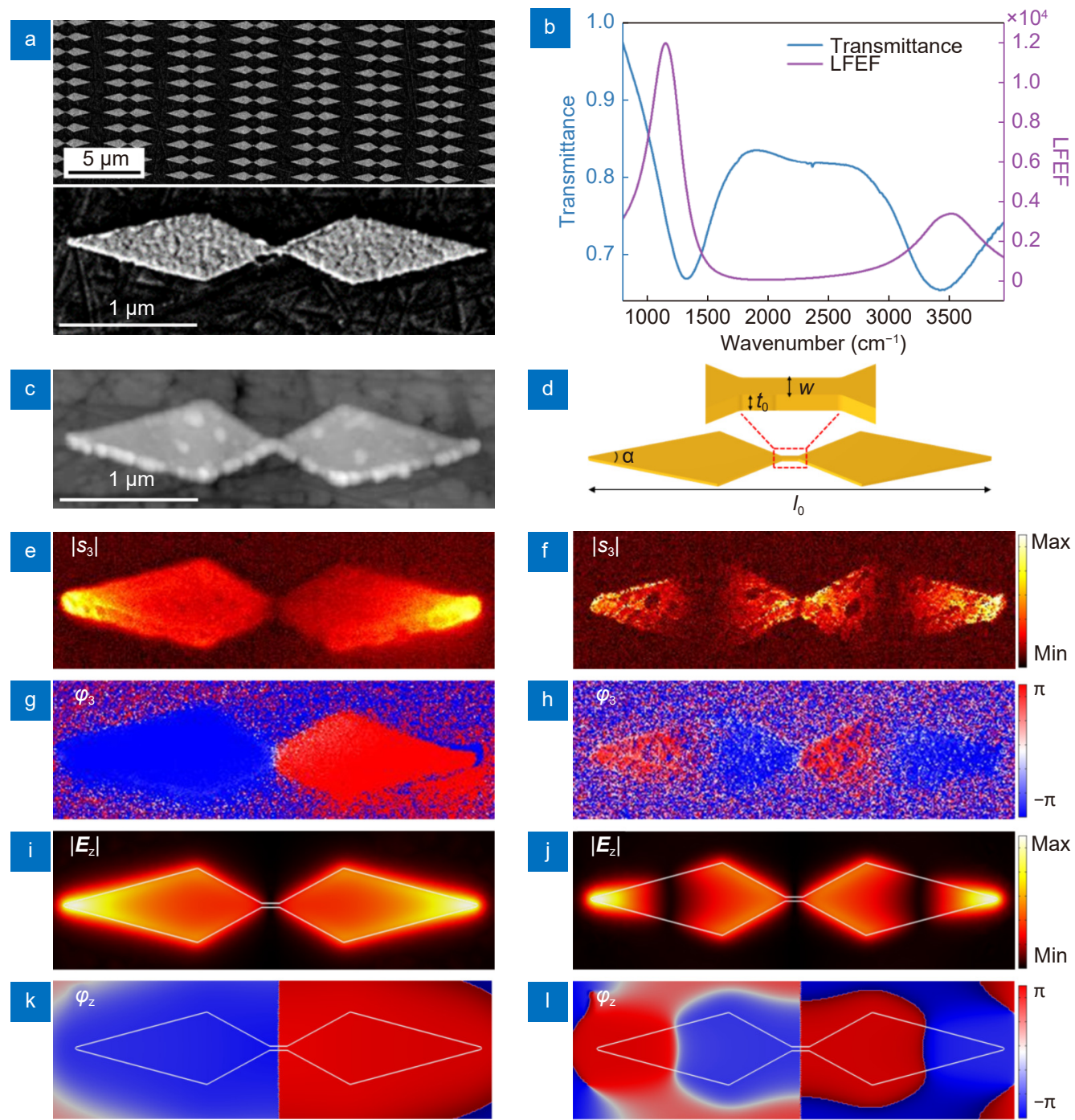

Fig. 1 (a) Scanning electron microscopy (SEM) image of NBRA structures. (b) Experimental transmittance of the NBRA array (blue curve) and simulated LFEF at the extremities of the structure (purple curve). (c) AFM topography of a single NBRA structure. The scale bars are $1 \mu$ m. (d) Sketch and parameters of the NBRA structure, consisting of two rhombic arms connected with each other by a nanobridge. The thickness ( $t_{0}$ ) and total length $\left(I_{0}\right)$ of NBRA are $30 \mathrm{~nm}$ and $2800 \mathrm{~nm}$. The nanobridge is $30 \mathrm{~nm}$ in width and $130 \mathrm{~nm}$ in length. The radius of sharp tip of each arm is $10 \mathrm{~nm}$ and $\alpha$ is $30^{\circ}$. (e-h) Measured near-field $(\mathrm{e}, \mathrm{f})$ amplitude and $(\mathrm{g}, \mathrm{h})$ phase of single NBRA structure at $1100 \mathrm{~cm}^{-1}$ and $2100 \mathrm{~cm}^{-1}$, respectively. (i-l) Simulated $\left|E_{\mathrm{z}}\right|(\mathrm{i}, \mathrm{j})$ and $\varphi_{\mathrm{z}}(\mathrm{k}, \mathrm{l})$ of single NBRA structure at $1100 \mathrm{~cm}^{-1}$ and $2100 \mathrm{~cm}^{-1}$, respectively. 
well as the simulated LFEF (Fig. 1(b)), show that the NBRA supports two pronounced resonance bands. One band is centered at $1319 \mathrm{~cm}^{-1}$, the other is centered at $3425 \mathrm{~cm}^{-1}$. The simulated transmittance spectra also show that the resonant absorption of the NBRA structure in the mid-infrared range only occurs when the polarization is along the long axis of the NBRA (Fig. S5, Supplementary information). To assign the plasmonic modes associated with the two bands, we employed the s-SNOM with the pseudo-heterodyne interferometric detection module which can provide almost background-free near-field amplitude and phase (see Methods for details $)^{46,47,55}$, to carry out the near-field imaging of the NBRA. Due to the limited output range of the QCL source in our laboratory, we choose $1100 \mathrm{~cm}^{-1}$ and $2100 \mathrm{~cm}^{-1}$ for the imaging of each band.

The measured near-field amplitude image exhibits two hotspots at the two extremities of the structure (Fig. 1(e)) when illuminating with a continuum-wave beam at 1100 $\mathrm{cm}^{-1}$ from the QCL, which is in accord with the simulated $\left|\boldsymbol{E}_{z}\right|$ (Fig. 1(i)). Parallelly, the measured near-field phase image exhibits a phase jump of $\sim \pi$ at the center of the nanobridge (Fig. 1(g)), in accord with the simulated phase image $\varphi_{z}$ (Fig. $1(\mathrm{k})$ ), and provides direct experimental evidence for the anti-phase field oscillating at $1100 \mathrm{~cm}^{-1}$. This mode was assigned to the charge-transfer plasmon (CTP) mode ${ }^{56-58}$, in which the current density is mainly confined at the nanobridge (Fig. S6(a), Supplementary information). The CTP mode is different from the dipolar mode of a single-arm antenna in which the current density is distributed along the antenna except for the two ends (Fig. S6(c), Supplementary information).

To assign the plasmonic mode associated with the band centered at $3425 \mathrm{~cm}^{-1}$, we performed the s-SNOM imaging at $2100 \mathrm{~cm}^{-1}(4.76 \mu \mathrm{m})$ which corresponds to the shortest wavelength of the QCL source in our laboratory. The simulated LFEF at $2100 \mathrm{~cm}^{-1}$ is around 25, which means the s-SNOM signals would be rather weak. In spite of this, the simulated charge distribution (Fig. S7, Supplementary information) shows similar characteristic between $2100 \mathrm{~cm}^{-1}$ and $3425 \mathrm{~cm}^{-1}$. Thus the sSNOM imaging still allows us to distinguish the plasmonic mode of this band. The measured near-field amplitude (Fig. 1(f)) and the simulated $\left|\boldsymbol{E}_{\mathrm{z}}\right|$ (Fig. 1(j)) show relatively strong signals at the two extremities of each rhombic arm as well. Notably, the measured near-field phase (Fig. 1(h)) and simulated $\varphi_{\mathrm{Z}}$ (Fig. 1(1)) show phase jumps of $\sim \pi$ at three locations: the center of each rhombic arm and the center of the nanobridge. This mode looks like two dipoles coupled through the nanobridge, and thus was named as the bridged dipolar plasmon (BDP) mode in which the current density is mainly confined at the nanobridge (Fig. S6(b), Supplementary information). Moreover, the main hotspots of both bands (Fig. 1(e), 1(f) and Fig. S8, Supplementary information) are spatially superimposed at the extremities of NBRA structure, where the LFEFs are $\sim 1.3 \times 10^{4}$ for the CTP and $\sim 3.4 \times 10^{3}$ for the BDP according to the electromagnetic simulations (Fig. 1(b)).

The NBRA excites the high-order mode more efficiently than the nanobar. The extinction spectra of the NBRA and the nanobar monomer both show two resonant bands in the mid-infrared range (Fig. S9(a), Supplementary information). The extinction intensities of the NBRA at the first and second band are $8.3 \%$ and $156.1 \%$ larger than those of the nanobar (Fig. S9(a), Supplementary information). Meanwhile, the resonant wavenumbers of the NBRA are slightly red-shift compared with that of the nanobar. And the full width at half maximum (FWHM) of the resonant bands of the NBRA are broader than those of the nanobar (Table S2, Supplementary information).

Comparing with other nanobridged structures, such as nanobridged-disks (Fig. 2(b)) or rectangles (Fig. 2(c)), the NBRA (Fig. 2(a)) shows distinct multiband resonances in the mid-infrared region in the simulated extinction spectra (Fig. 2(d)). Further, the hotspots of the NBRA are located at the extremities of the structure (Fig. 2(f)), while the hotspots of nanobridged-disks or rectangles at the CTP resonance are distributed dispersively (Fig. 2(g) and 2(h)), resulting to ten times lower LFEFs than that of the NBRA (Fig. 2(e)).

The CTP and BDP bands of the NBRA are strongly associated with the geometrical parameters of the nanobridge. Figure 3(a) shows that the CTP band redshifts and weakens, and the BDP band redshifts and broadens as the width of the nanobridge decreases from $30 \mathrm{~nm}$ to 5 $\mathrm{nm}$. As the nanobridge is broken and transforms to a nanogap in between the two arms, the BDP band disappears in their stead a bonding dipolar plasmonic mode (Fig. 3(b)) and a bonding quadrupolar plasmonic mode (Fig. 3(c)) appears. In contrast, as the width of the nanobridge increases from $30 \mathrm{~nm}$ to $370 \mathrm{~nm}$, the CTP band blueshifts and strengthens, and the BDP band blueshifts and weakens. Ultimately, the CTP and BDP bands evolve 

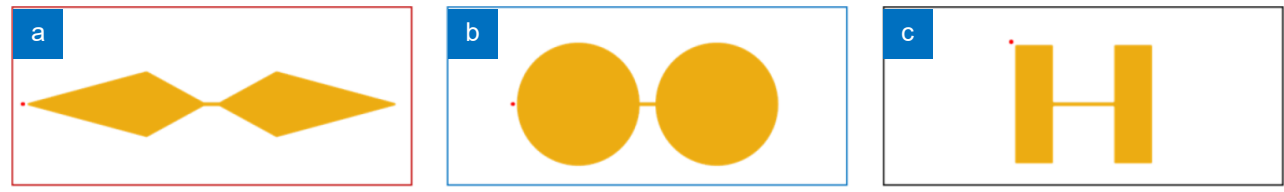

d

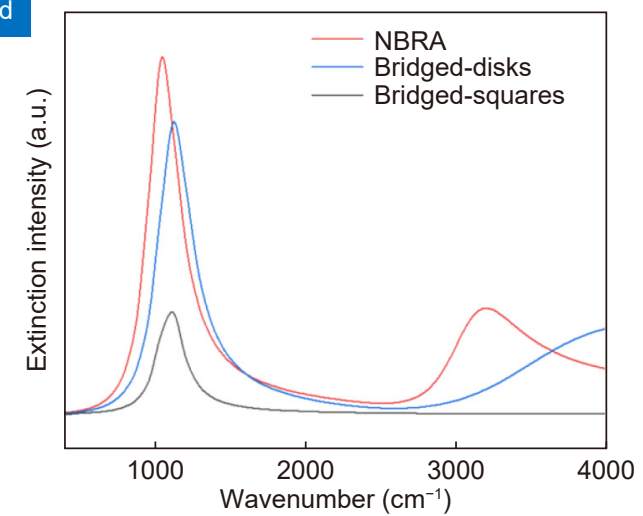

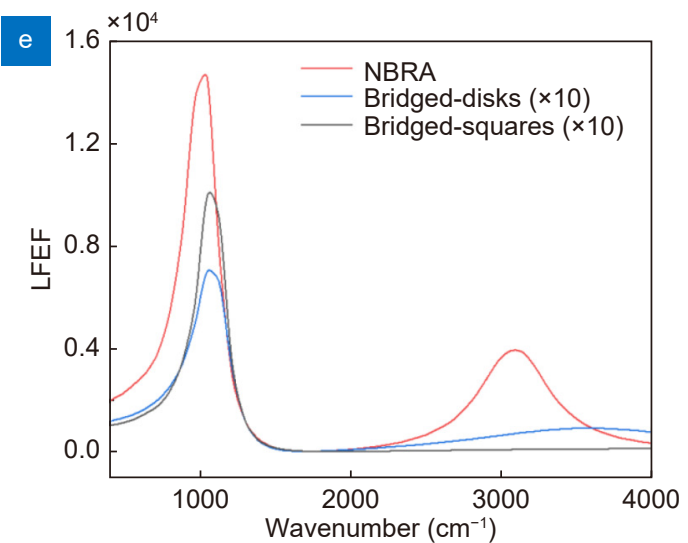
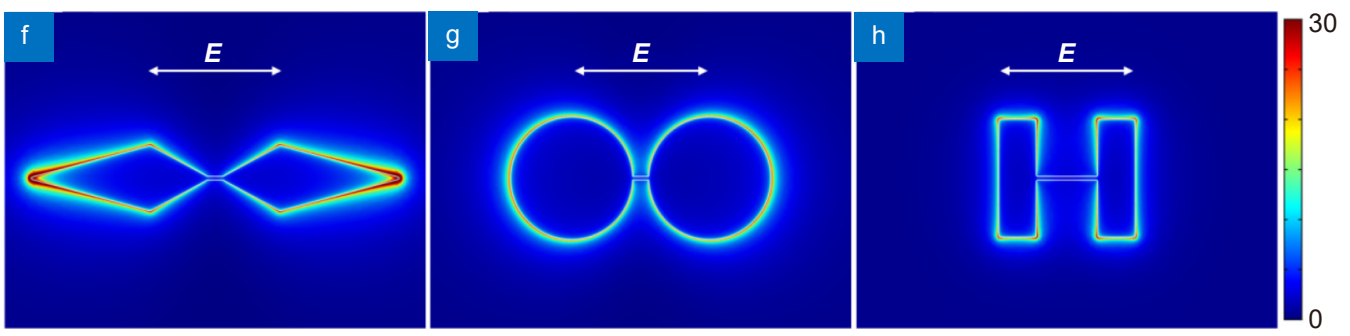

Fig. 2 | The model of (a) NBRA, (b) nano-bridged disks and (c) nano-bridged rectangles. All the structures are placed on the CaF 2 substrate. The incident polarizations are along the long-axis of the nanobridge. The thickness and the width of the nanobridge are $30 \mathrm{~nm}$ for all three structures. The lengths of the nanobridges are $130 \mathrm{~nm}$ for the NBRA and the nanobridged-disks, and $500 \mathrm{~nm}$ for the nanobridged-rectangles. The total length of the NBRA is $3000 \mathrm{~nm}$. The radius of the disks are $500 \mathrm{~nm}$. The length and width of the rectangles are $962 \mathrm{~nm}$ and $308 \mathrm{~nm}$, respectively. The red points in $(\mathrm{a}-\mathrm{c})$ are the positions where the LFEFs are evaluated. The LFEF was evaluated at the point $2 \mathrm{~nm}$ away from the structure along the long axis and $15 \mathrm{~nm}$ above the $\mathrm{CaF}_{2}$ substrate for the NBRA and the nanobridged-disks. While for the nanobridged-rectangles, the evaluating point is $2 \mathrm{~nm}$ away from the corner of the rectangle. (d) The simulated extinction spectra and (e) the LFEFs of all three structures. Efield distributions at the CTP resonance for (f) the NBRA, (g) the nanobridged-disks and (h) the nanobridged-rectangles.

to a typically dipolar band (Fig. 3(d)) and quadrupolar band (Fig. 3(e)), respectively, similar to that in a nanobar antenna of the same length (Fig. S10, Supplementary information).

An circuit model consisting of resistances (R), inductances (L) and capacitors (C) was built to understand the role of the nanobridge width-tuned plasmonic resonant frequencies and extinction intensities. As shown in the inset of Fig. 4(a), each rhombic arm as well as the nanobridge was modelled as a parallel RLC circuit, and all of three circuits were further connected in series. The total impedance of the circuits system is:

$$
Z(\omega)=\frac{\omega L_{\mathrm{b}} R_{\mathrm{b}}}{\omega L_{\mathrm{b}}+\mathrm{i} R_{\mathrm{b}}\left(1-\omega^{2} L_{\mathrm{b}} C_{\mathrm{b}}\right)}+\frac{2 \omega L_{0} R_{0}}{\omega L_{0}+\mathrm{i} R_{0}\left(1-\omega^{2} L_{0} C_{0}\right)} .
$$

The extinction spectrum is proportional to the real part of $Z^{59}$.

$$
\begin{aligned}
R e(Z(\omega)) & =\frac{\omega^{2} L_{\mathrm{b}}^{2} R_{\mathrm{b}}}{R_{\mathrm{b}}^{2} L_{\mathrm{b}}^{2} C_{\mathrm{b}}^{2} \omega^{4}+\omega^{2}\left(L_{\mathrm{b}}^{2}-2 R_{\mathrm{b}}^{2} L_{\mathrm{b}} C_{\mathrm{b}}\right)+R_{\mathrm{b}}^{2}} \\
& +\frac{2 \omega^{2} L_{0}^{2} R_{0}}{R_{0}^{2} L_{0}^{2} C_{0}^{2} \omega^{4}+\omega^{2}\left(L_{0}^{2}-2 R_{0}^{2} L_{0} C_{0}\right)+R_{0}^{2}},
\end{aligned}
$$

where $R_{\mathrm{b}}, L_{\mathrm{b}}$ and $C_{\mathrm{b}}$ are the resistance, inductance and capacitance of the nanobridge and all depend on the width of the nanobridge; $R_{0}, L_{0}$ and $C_{0}$ are the resistance, inductance and capacitance of each rhombic arm and are also all dependent on the nanobridge width since the geometric cross section of one end of each rhombic arm and that of the linked nanobridge are the same.

Herein, $L_{\mathrm{b}}$ is the sum of conventional magnetic inductance $L_{\mathrm{M}} \sim \frac{\mu_{0} l_{0}}{2 \pi} \ln {\frac{l_{0}}{w+h}}^{57,59,60}$, and the kinetic inductance $L_{\mathrm{K}}={\frac{l_{0}}{w h \varepsilon_{0} \omega_{p}^{2}}}^{61,62}$, where $\varepsilon_{0}$ and $\mu_{0}$ are, respectively, the permittivity and the permeability of free space, $l_{0}, w$ and $h$ are, respectively, the length, width, and height of 

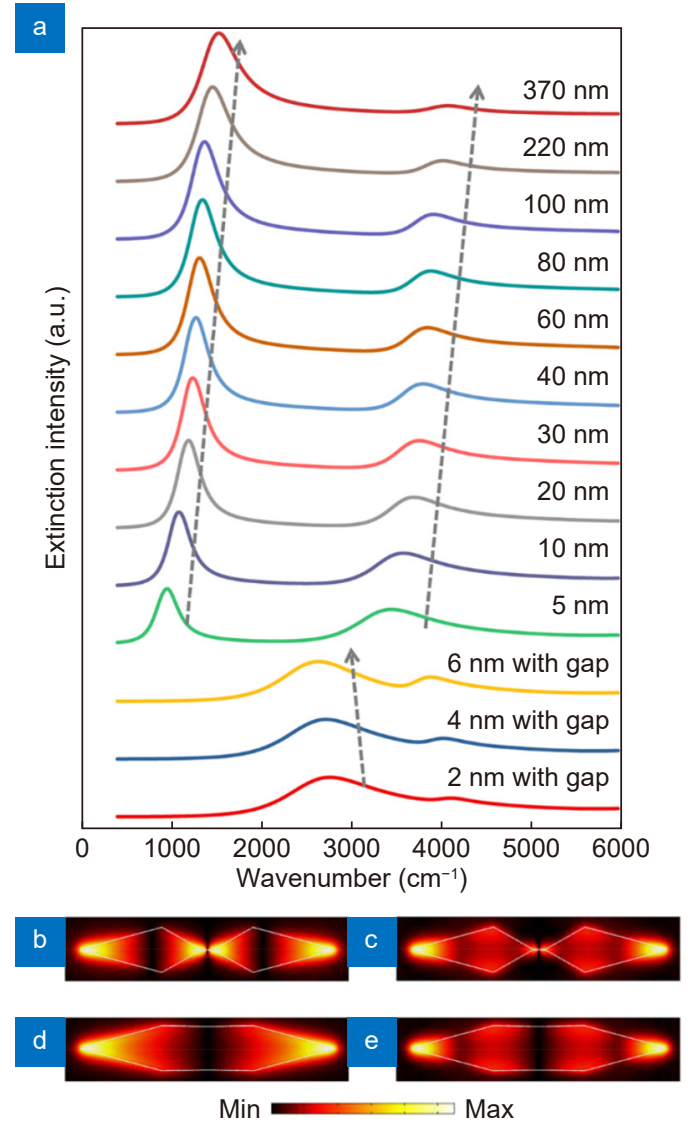

Fig. 3 | (a) Simulated extinction spectra of NBRA structure with different nanobridge width. (b-c) Simulated mapping of $\left|E_{\mathrm{z}}\right|$ of bonding dipolar plasmonic band (b) and bonding quadrupolar plasmonic band (c) of a single NBRA structure with a broken nanobridge and $4 \mathrm{~nm}$ gap size. (d-e) Simulated mapping of $\left|E_{z}\right|$ at CTP band (d) and BDP band (e) with $370 \mathrm{~nm}$ nanobridge width.

the nanobridge; and $\omega_{p}$ is the plasma frequency of the metal. Thus, $L_{\mathrm{b}}$ can be written as $L_{\mathrm{b}} \sim\left(\frac{a_{1}}{w}+a_{2} \ln \frac{a_{3}}{w}\right)$, where $a_{1}, a_{2}$ and $a_{3}$ are fitting parameters. The inductance of the rhombic arms obeys the same expression as $L_{0} \sim\left(\frac{b_{1}}{w}+b_{2} \ln \frac{b_{3}}{w}\right) . C_{\mathrm{b}}$ and $C_{0}$ linearly depend on the nanobridge $\quad$ width $^{59,60}, \quad C_{\mathrm{b}} \sim\left(c_{1} w+c_{2}\right) \quad$ and $C_{0} \sim\left(d_{1} w+d_{2}\right)$.

In this study, we considered the essential impact of $R_{\mathrm{b}}$ and $R_{0}$ on the extinction intensities of both bands, which have not been considered in the previous study ${ }^{56}$. Taking the featured geometries of the NBRA structure, we derived the $w$-dependent $R_{\mathrm{b}}$ and $R_{0}$ :

$$
\begin{gathered}
R_{\mathrm{b}} \sim\left(\frac{e_{1}}{e_{2}-w} \ln \frac{e_{2}}{w}+\frac{e_{3}}{w}\right), \\
R_{0} \sim\left(\frac{f_{1}}{f_{2}-w} \ln \frac{f_{2}}{w}+\frac{f_{3}}{w}+f_{4}\right) .
\end{gathered}
$$

The detailed derivations of $R_{\mathrm{b}}$ and $R_{0}$ as a function of nanobridge width are described in Supporting Information section 1 . The aforementioned $b_{1,2,3}, c_{1,2}, d_{1,2} e_{1,2,3}$, and $f_{1,2,3,4}$ are all fitting parameters.

Fitting $\operatorname{Re}(Z(\omega))$ containing all 17 fitting parameters in a one-step fit is too complicated to be realized. Instead, we used a two-step fit to obtain all the fitting parameters, as shown in Table 1. The detailed fitting procedures are described in Supporting Information section 2. Consequently, we got the $w$-dependent $L_{\mathrm{b}}, L_{0}, C_{\mathrm{b}}, C_{0}, R_{\mathrm{b}}$ and $R_{0}$ curves (Fig. S11, Supplementary information) and $\operatorname{Re}(Z(\omega))$ spectra (Fig. S12, Supplementary information). Fig. 4(a) and 4(b) shows that the $w$-dependent resonant frequencies and intensities which were extracted from the two dominant bands in the $w$-dependent spectra of $\operatorname{Re}(Z(\omega))$ (Fig. S12, Supplementary information) match well with the $w$-dependent resonant frequencies and extinction intensities of the CTP and BDP bands which were extracted from the simulated extinction spectra (Fig. 3(a)).

The analytical form of $Z(\omega)$ enables us to explore the main factors affecting the resonant frequencies. Typically, the resonant frequencies of an RLC circuit make the imaginary part of $Z(\omega)$, as shown in Eq. (5), be zero.

$$
\begin{aligned}
\operatorname{Im}(Z(\omega)) & =\frac{\omega R_{\mathrm{b}}^{2} L_{\mathrm{b}}\left(L_{\mathrm{b}} C_{\mathrm{b}} \omega^{2}-1\right)}{R_{\mathrm{b}}^{2} L_{\mathrm{b}}^{2} C_{\mathrm{b}}^{2} \omega^{4}+\omega^{2}\left(L_{\mathrm{b}}^{2}-2 R_{\mathrm{b}}^{2} L_{\mathrm{b}} C_{\mathrm{b}}\right)+R_{\mathrm{b}}^{2}} \\
& +\frac{2 \omega R_{0}^{2} L_{0}\left(L_{0} C_{0} \omega^{2}-1\right)}{R_{0}^{2} L_{0}^{2} C_{0}^{2} \omega^{4}+\omega^{2}\left(L_{0}^{2}-2 R_{0}^{2} L_{0} C_{0}\right)+R_{0}^{2}} .
\end{aligned}
$$

However, the exact solutions to Eq. (5) are too complicated to be further analyzed. Instead, we adopted commonly used simplification, independently making the first term or the second term of Eq. (5) be zero. Then we got the simplified solutions:

$$
\begin{aligned}
& \omega_{1}=\frac{1}{\sqrt{L_{\mathrm{b}} C_{\mathrm{b}}}} . \\
& \omega_{2}=\frac{1}{\sqrt{L_{0} C_{0}}} .
\end{aligned}
$$

It can be found that, $\omega_{1}$ and $\omega_{2}$ correspond to the RLC resonances of the independent nanobridge and rhombic arm respectively. Furthermore, Eqs. $(6,7)$ are good approximations for $\omega_{\mathrm{CTP}}$ and $\omega_{\mathrm{BDP}}$, because the deviations between $\omega_{1,2}$ and $\omega_{\text {CTP }, \text { BDP }}$ extracted from the $\operatorname{Re}(Z(\omega))$ spectra are less than 1\%, as shown in Fig. S13 (see Supplementary information).

$$
\omega_{\mathrm{CTP}} \approx \frac{1}{\sqrt{L_{\mathrm{b}} C_{\mathrm{b}}}},
$$



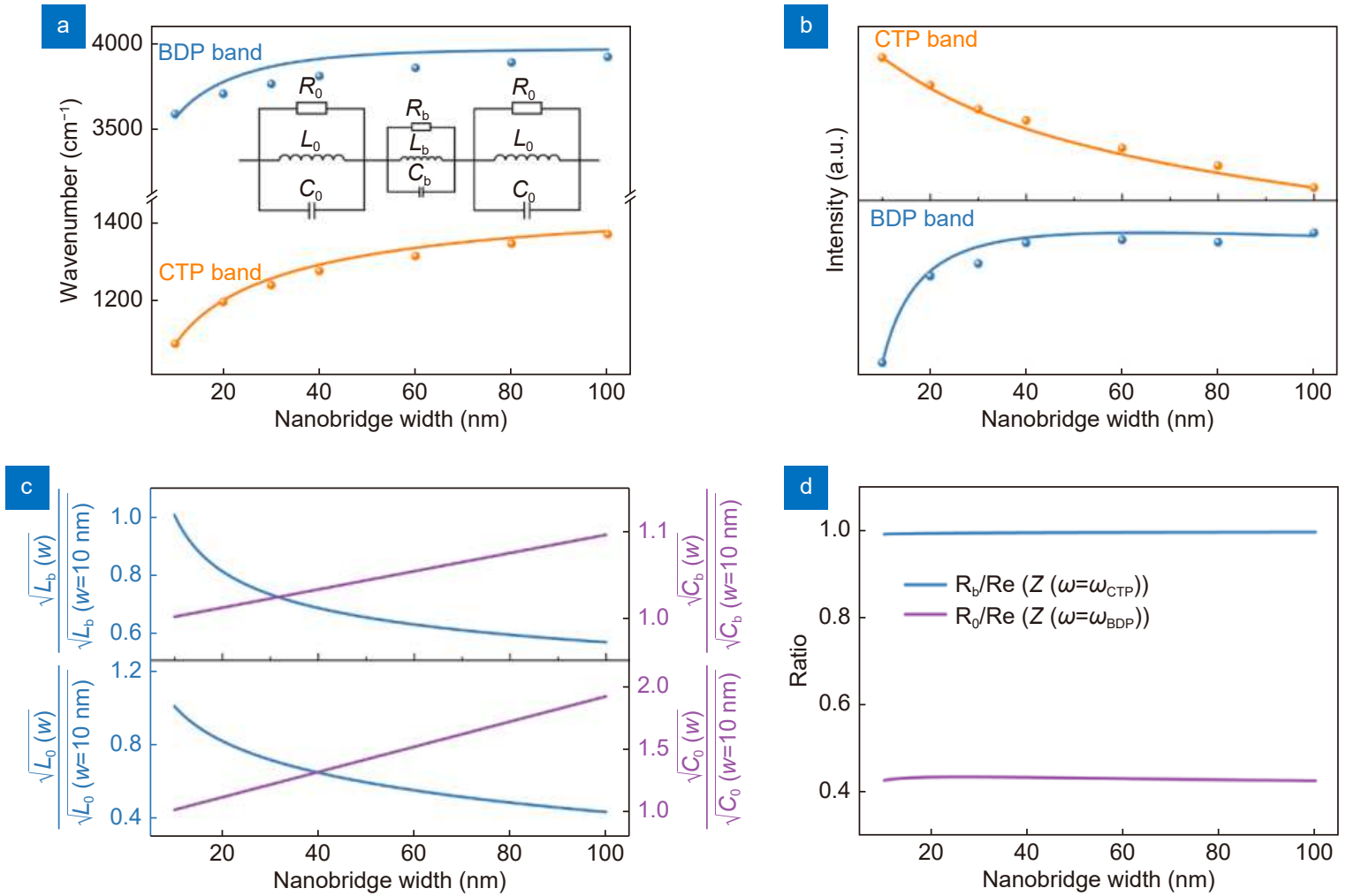

Fig. 4 | Nanobridge width-dependent (a) resonant frequencies and (b) intensities of CTP and BDP bands. The orange and blue circles are extracted from the simulated extinction spectra (Fig. 3(a)), while the points on the fitting curves are extracted from the spectra of Re (Z( $\omega)$ ) (Fig. S12, Supplementary information). Inset in (a): RLC circuit model of the NBRA structure. (c) Nanobridge width-dependent ratio of $\frac{\sqrt{L_{\mathrm{b}}(w)}}{\sqrt{L_{\mathrm{b}}(w=10 \mathrm{~nm})}}$ (blue curve in the top panel), $\frac{\sqrt{C_{\mathrm{b}}(w)}}{\sqrt{C_{\mathrm{b}}(w=10 \mathrm{~nm})}}$ (purple curve in the top panel), $\frac{\sqrt{L_{0}(w)}}{\sqrt{L_{0}(w=10 \mathrm{~nm})}}$ (blue curve in the bottom panel) and $\frac{\sqrt{C_{0}(w)}}{\sqrt{C_{0}(w=10 \mathrm{~nm})}}$ (purple curve in the bottom panel). (d) Nanobridge width-dependent ratio of $\frac{R_{\mathrm{b}}}{\operatorname{Re}\left(Z\left(\omega=\omega_{\mathrm{CTP}}\right)\right)}$ (blue curve) and $\frac{R_{0}}{\operatorname{Re}\left(Z\left(\omega=\omega_{\mathrm{BDP}}\right)\right)}$ (purple curve). $R_{b}$ and $R_{0}$ are the deterministic parameters for $\operatorname{Re}(Z(\omega)$.

Table 1 | Fitting parameters used in the RCL circuit model

\begin{tabular}{|c|c|c|c|}
\hline Parameter & Value & Parameter & Value \\
\hline$a_{1}$ & $2.17 \times 10^{-10}$ & $d_{1}$ & $3.82 \times 10^{-19}$ \\
\hline$a_{2}$ & $6.75 \times 10^{-12}$ & $d_{2}$ & $3.39 \times 10^{-17}$ \\
\hline$a_{3}$ & $5.74 \times 10^{4}$ & $e_{1}$ & $9.77 \times 10^{11}$ \\
\hline$b_{1}$ & $1.64 \times 10^{-12}$ & $e_{2}$ & -7184.74 \\
\hline$b_{2}$ & $5.14 \times 10^{-13}$ & $e_{3}$ & $3.21 \times 10^{5}$ \\
\hline$b_{3}$ & 650.03 & $f_{1}$ & 1146.59 \\
\hline$c_{1}$ & $1.24 \times 10^{-20}$ & $f_{2}$ & -2232.04 \\
\hline$c_{2}$ & $1.18 \times 10^{-17}$ & $f_{3}$ & 1 \\
\hline
\end{tabular}

$$
\omega_{\mathrm{BDP}} \approx \frac{1}{\sqrt{L_{0} C_{0}}}
$$

The inductances $L_{\mathrm{b}}$ and $L_{0}$ are the determining quantities for $\omega_{\text {CTP }}$ and $\omega_{\mathrm{BDP}}$ as a function of $w . L_{\mathrm{b}}$ and $L_{0}$ decrease (Fig. S11(a, b), Supplementary information), while the $\frac{1}{\sqrt{L_{\mathrm{b}}}}$ and $\frac{1}{\sqrt{L_{0}}}$ increase (Fig. 4(c)) as the nanobridge becomes wider, which is consistent with the blue shift of
$\omega_{\mathrm{CTP}}$ and $\omega_{\mathrm{BDP}}($ Fig. $4(\mathrm{a}))$. Contrarily, the $\frac{1}{\sqrt{C_{\mathrm{b}}}}$ and $\frac{1}{\sqrt{C_{0}}}$ decrease as $w$ increases (Fig. $4(\mathrm{c})$ ). Furthermore, $\frac{1}{\sqrt{L_{\mathrm{b}}}}$ and $\frac{1}{\sqrt{L_{0}}}$ increase more than $10 \%$ and $90 \%$ respectively while $\frac{1}{\sqrt{C_{\mathrm{b}}}}$ and $\frac{1}{\sqrt{C_{0}}}$ both decrease around $50 \%$ as $w$ increases from $10 \mathrm{~nm}$ to $100 \mathrm{~nm}$. 
To study the key quantities to determine the extinction intensities (Fig. 4(b)) of both bands $\left(\sim \operatorname{Re}\left(Z\left(\omega=\omega_{\text {СTP }}, \omega_{\text {BDP }}\right)\right)\right)$, we substituted Eqs. (8) and (9) into Eq. (2), and got,

$$
\operatorname{Re}\left(Z\left(\omega=\omega_{\text {CTP }}\right)\right)=\frac{2 L_{0}^{2} L_{\mathrm{b}} C_{\mathrm{b}} R_{0}}{R_{0}^{2}\left(L_{0} C_{0}-L_{\mathrm{b}} C_{\mathrm{b}}\right)^{2}+L_{0}^{2} L_{\mathrm{b}} C_{\mathrm{b}}}+R_{\mathrm{b}},
$$

$\operatorname{Re}\left(Z\left(\omega=\omega_{\mathrm{BDP}}\right)\right)=\frac{2 L_{\mathrm{b}}^{2} L_{0} C_{0} R_{\mathrm{b}}}{R_{\mathrm{b}}^{2}\left(L_{\mathrm{b}} C_{\mathrm{b}}-L_{0} C_{0}\right)^{2}+L_{\mathrm{b}}^{2} L_{0} C_{0}}+2 R_{0}$

Figure 4(d) shows that $R_{\mathrm{b}}$ and $R_{0}$, are, respectively, the dominant terms of $\operatorname{Re}\left(Z\left(\omega=\omega_{\text {CTP }}\right)\right)$ and $\operatorname{Re}\left(Z\left(\omega=\omega_{\mathrm{BDP}}\right)\right)$. As $w$ becomes wider, $R_{\mathrm{b}}$ increases and $R_{0}$ decreases (Fig. S11(e, f), Supplementary information), resulting to the strengthening of the CTP band and the weakening of the BDP band. In brief, the resonant frequencies and extinction intensities of both bands are strongly dependent on the nanobridge width which simultaneously determines the inductance and resistance of the nanobridge and the rhombic arms.

\section{Tunability of MIR resonance and SEIRA}

The resonant wavelengths of the two bands can be tuned not only by varying the width of nanobridge, but also by varying the total length $l_{0}$ of NBRAs (Fig. 5(a) and Fig. S14(a), Supplementary information), just as what is observed for the rods structures ${ }^{24-26}$. The data between 2000 2600 $\mathrm{cm}^{-1}$ is hided in Fig. 5(a) to highlight the resonant dips of both bands. Experimentally, the resonant wavelengths of the two bands, $\lambda_{\text {СТP }}$ and $\lambda_{\mathrm{BDP}}$, redshift linearly as the length of NBRA $l_{0}$ increases with fitting functions $\lambda_{\text {СTP }}=2.14 \times l_{0}+1.93(\mu \mathrm{m})$ for CTP band, and $\lambda_{\mathrm{BDP}}=0.78 \times l_{0}+0.86(\mu \mathrm{m})$ for BDP band (Fig. S14(b, c), Supplementary information). The tunability can be

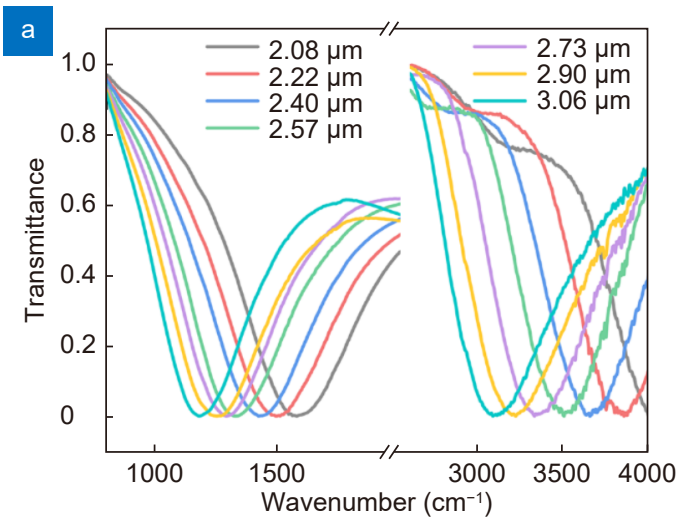

further evidenced by antenna length-tuned Fano interference between molecular vibrational excitation and plasmon excitation ${ }^{24,63}$. In our experiment, we functionalized a monolayer PNTP molecules whose symmetric stretching vibrational band of $\mathrm{NO}_{2}$ is centered at 1335 $\mathrm{cm}^{-1}$. As shown in Fig. 5(b), the lineshape of the band around $1335 \mathrm{~cm}^{-1}$ of the measured SEIRA spectra evolves from an asymmetric Fano shape to a Lorentzian shape, and to an asymmetric Fano shape as the resonant wavenumber of the CTP band increases from $1176 \mathrm{~cm}^{-1}$ to $1496 \mathrm{~cm}^{-1}$. For the BDP band covering the $=\mathrm{C}-\mathrm{H}$ stretching band of PNTP at $3024 \mathrm{~cm}^{-1}$, the SEIRA spectra around $3024 \mathrm{~cm}^{-1}$ barely show a signal due to the weakness of the LFEF. Comparing with $w, l_{0}$ is less efficient when modifying the resonant wavelengths of the NBRA. As $w$ increases $365 \mathrm{~nm}$ (from $5 \mathrm{~nm}$ to $370 \mathrm{~nm}$ ), the resonant wavelengths of the CTP band and BDP band blue shift about $3.9 \mu \mathrm{m}$ and $0.45 \mu \mathrm{m}$. However, as $l_{0}$ increases nearly $1 \mu \mathrm{m}$ (from $2.08 \mu \mathrm{m}$ to $3.06 \mu \mathrm{m}$ ), the resonant wavelengths of the CTP band and BDP band only red shift about $2.01 \mu \mathrm{m}$ and $0.76 \mu \mathrm{m}$.

\section{Nanogaped NBRA dimer}

The spatially superimposed hotspots at the two extremities of the NBRA structure pave the way for further boosting up the local field for both bands in a NBRA dimer with a nanometer-size gap (nanogap). The NBRA dimer was fabricated with a $\sim 20 \mathrm{~nm}$ gap size (see the SEM image and AFM topography in Fig. 6(a), 6(c) and Fig. S3(b), Supplementary information)). Other geometric parameters are the same as those of the structure in Fig. 1. The periodicities of the array are $6400 \mathrm{~nm}$ and 1000 $\mathrm{nm}$ along the long and short axes of the NBRA dimer, respectively. The normalized experimental transmittance spectra of NBRA dimers also exhibit two pro-

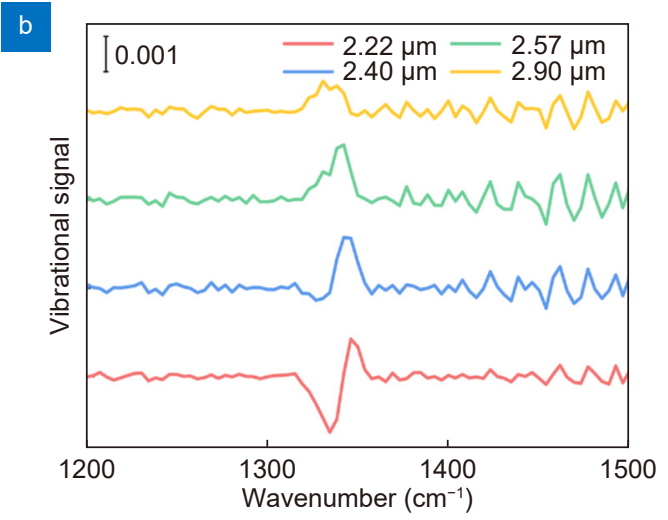

Fig. 5 | (a) Experimental transmittance spectra (normalized) as a function of the length of the NBRAs. (b) SEIRA spectra of PNTP on NBRAs with total lengths 2.22, 2.40, 2.57 and $2.90 \mu \mathrm{m}$ in the range of the CTP band. 

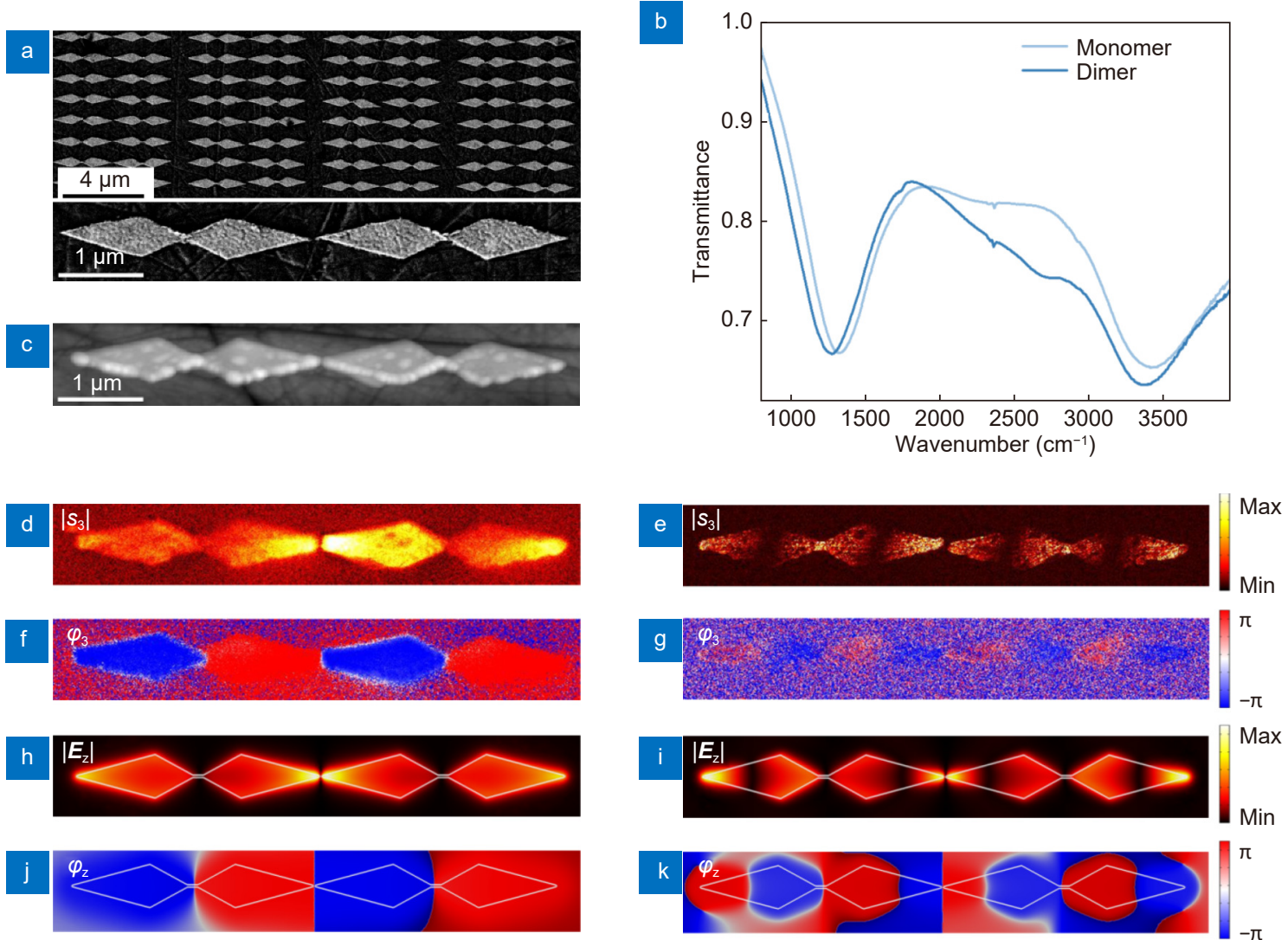

Fig. 6 | (a) SEM image of NBRA dimer. (b) Experimental transmittance of a NBRA and its dimer with gap size $20 \mathrm{~nm}$. (c) AFM topography of a NBRA dimer. The scale bars in (a) and (c) are $1 \mu \mathrm{m}$. Measured near-field amplitude (d) and phase (f) of NBRA dimer at $1100 \mathrm{~cm}^{-1}$. Simulated mapping of $\left|E_{\mathrm{z}}\right|(\mathbf{h})$ and $\varphi_{\mathrm{z}}(\mathbf{j})$ of NBRA dimer at $1100 \mathrm{~cm}^{-1}$. Measured near-field amplitude $(\mathbf{e})$ and phase $(\mathbf{g})$ of NBRA dimer at $2100 \mathrm{~cm}^{-1}$. Simulated mapping of $\left|E_{\mathrm{z}}\right|(\mathbf{i})$ and $\varphi_{\mathrm{z}}(\mathbf{k})$ of NBRA dimer at $2100 \mathrm{~cm}^{-1}$.

nounced resonance bands in MIR spectral range, one band centered at $1272 \mathrm{~cm}^{-1}$ and the other one centered at $3367 \mathrm{~cm}^{-1}$ (Fig. 6(b)). Both bands of NBRA dimer only redshift less than $100 \mathrm{~cm}^{-1}$ in comparison with the monomer counterparts (Fig. 6(b)). To assign the plasmonic modes associated with the two bands, we employed the s-SNOM to measure the NBRA dimer for both bands. The near-field amplitude and phase images at $1100 \mathrm{~cm}^{-1}$, as shown in Fig. 6(d) and 6(f), as well as the simulated $\left|\boldsymbol{E}_{\mathrm{z}}\right|$ and $\varphi_{\mathrm{z}}$ shown in Fig. 6(h) and 6(j), suggest that the band centered at $1272 \mathrm{~cm}^{-1}$ is a gap-coupled CTP band of the NBRA-dimer structure. The near-field amplitude and phase images at $2100 \mathrm{~cm}^{-1}$, as shown in Fig. 6(e) and 6(g), as well as the simulated $\left|\boldsymbol{E}_{\mathrm{z}}\right|$ and $\varphi_{\mathrm{z}}$ shown in Fig. 6(i) and 6(k), suggest that the band centered at $3367 \mathrm{~cm}^{-1}$ can be assigned to a gap-coupled BDP band, although $2100 \mathrm{~cm}^{-1}$ is located at the shoulder of the $3367 \mathrm{~cm}^{-1}$ band. Notably, strong amplitude signals are found in the nanogap for both bands as expected ${ }^{47,64}$. The simulated LEEFs at the hotspots of both bands in the NBRA dimer are 6.3 and 5.0 times larger than those of the NBRA monomer. Further shrinking the nanogap size from $20 \mathrm{~nm}$ to $5 \mathrm{~nm}$, the LFEF can be boosted up about 20 times. Moreover, the logarithm of the EFs linearly depends on the gap size with the slope about -2 for both CTP and BDP bands (Fig. S16, Supplementary information), indicating that the near-field coupling through the nanogap for both bands in the NBRA dimer is attributed to the capacitive coupling ${ }^{28}$.

\section{NBRA dimer-waveguide-cavity coupling}

To further enhance LFEFs for both bands, we adopted a strategy by the optical coupling between a localized surface plasmon (LSP) mode and a waveguide-cavity mode ${ }^{14,65-67}$. As shown in Fig. 7(a), a NBRA dimer was fabricated on a reflective substrate (the NBRA dimer-onreflector structure). The reflective substrate was prepared by depositing $3 \mathrm{~nm}$ of Ti and $200 \mathrm{~nm}$ of Au onto a Si wafer using electron beam evaporation followed by depositing $3 \mathrm{~nm}$ of $\mathrm{Al}$ and $1400 \mathrm{~nm}$ of $\mathrm{SiO}_{2}$ using vacuum magnetron sputtering. Previous studies focused on a single LSP mode coupled with the waveguide cavity 

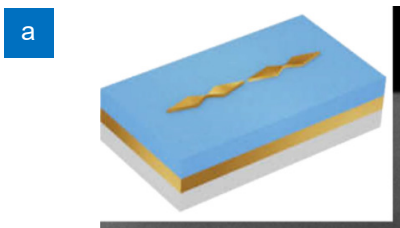

\section{$\mathrm{SiO}_{2}$ space layer}

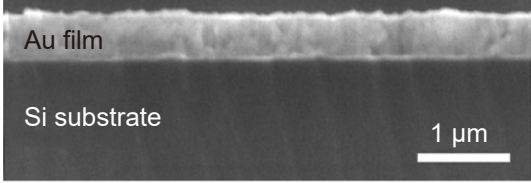

c

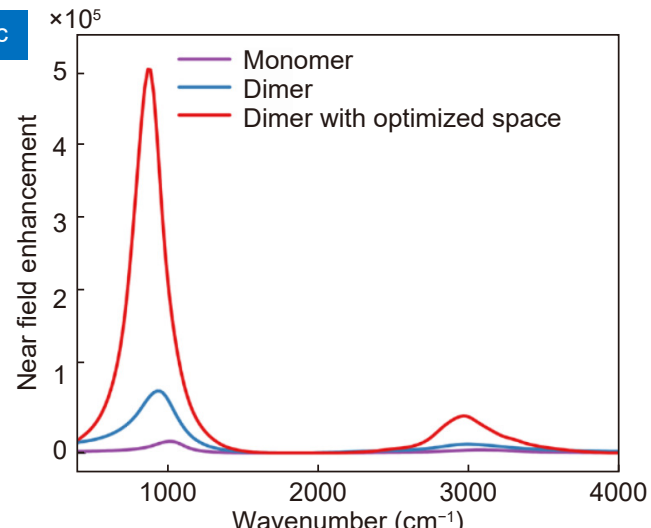

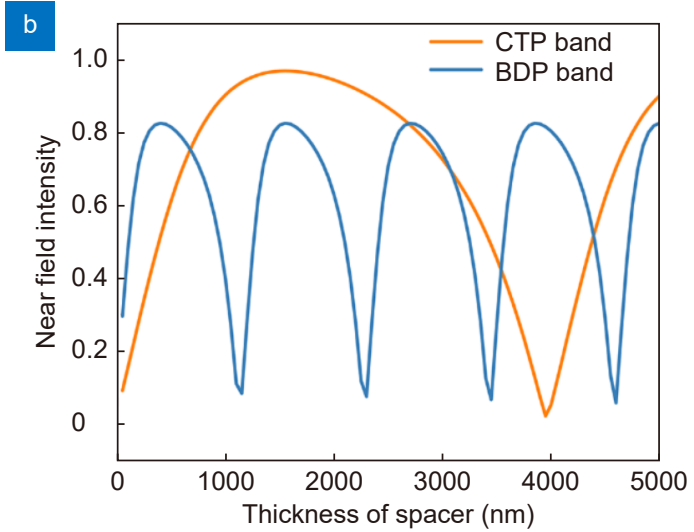

d

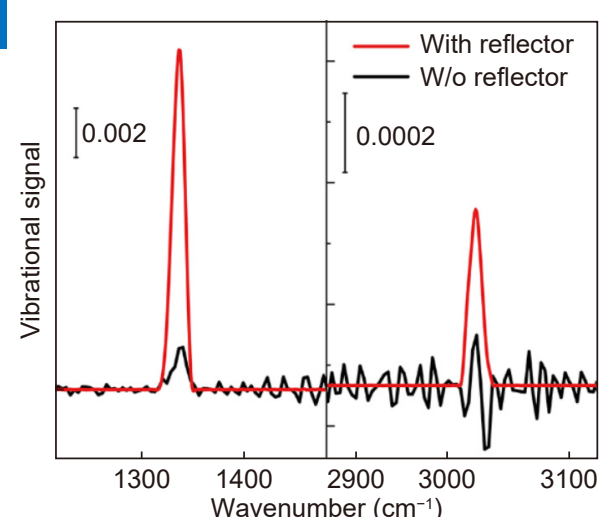

Fig. 7 | (a) SEM of the cross section of the reflective substrate. A $200 \mathrm{~nm}$ gold film is sandwiched between a $1400 \mathrm{~nm} \mathrm{SiO}_{2}$ spacer layer and the Si substrate. The scale bar is $1 \mu \mathrm{m}$. Inset: schematic of the NBRA dimer-on-reflector structure to integrate the waveguide-cavity coupling and nanogap coupling. (b) Normalized LFEFs of CTP mode (blue curve) and BDP mode (orange curve) as a function of the thickness of the spacer layer. (c) Simulated LFEFs of monomer, dimer and dimer with $1400 \mathrm{~nm} \mathrm{SiO} 2$ spacer layer. (d) SEIRA spectra of PNTP on NBRA dimer on transmitted substrate or on reflective substrate.

mode $^{14,67}$. In this study, we are targeting at both gapcoupled CTP and BDP bands rather than only one band of the NBRA that can be simultaneously and constructively coupled with the respective waveguide cavity modes on the same reflective substrate.

We numerically optimized the thickness of the spacer layer to simultaneously maximize the LFEFs in the nanogap of the NBRA dimer for both bands. Figure 7(b) shows that the simulated normalized LFEFs in the nanogap of the NBRA dimer-on-reflector structure for the gap-coupled CTP and BDP bands fluctuate periodically as the thickness of spacer layer $\left(\mathrm{SiO}_{2}\right)$ increases, with periodicities $4800 \mathrm{~nm}$ and $1150 \mathrm{~nm}$, respectively. The periodical behavior can be also predicted through the basic principles for electromagnetic waves ${ }^{68}$. We found that $1400 \mathrm{~nm}$ was the thinnest thickness of the $\mathrm{SiO}_{2}$ layer for almost simultaneously maximizing LFEFs in the nanogap of the NBRA dimer-on-reflector structure with additional 6.6 times and 4.0 times for the gap-coupled CTP and BDP bands, respectively (Fig. 7(c)), in compar- ison with those of the NBRA dimer without the reflector. Integrally, the LFEFs in the nanogap of the NBRA dimeron-reflector structure of both bands will be enhanced by 36.75 times and 12.35 times for CTP and BDP bands, respectively, compared with the NBRA monomer through nanogap coupling and waveguide-cavity coupling (Fig. 7(c)).

Experimentally, the SEIRA spectra of a monolayer of PNTP molecules on the surface of the NBRA dimer with or without the reflector structure (thickness of $\mathrm{SiO}_{2}$ spacer layer, $1400 \mathrm{~nm}$ ) further demonstrate the additional enhancement for both bands through waveguide-cavity coupling (Fig. 7(d)). The integral absorption intensities around $1335 \mathrm{~cm}^{-1}$ and $3024 \mathrm{~cm}^{-1}$ bands for the NBRA dimer-on-reflector structure are 7.4 times and 6.9 times larger than those of NBRA dimer without the reflector. The experimental results show that it is a practical strategy to boost the LFEFs associated with both bands by the waveguide-cavity coupling. 


\section{Conclusions}

In summary, we have reported a nanobridged rhombic structure as a new type of MIRA, effectively exciting the high-order mode (BDP mode) and the fundamental mode (CTP mode) through charge transfer plasmon, which has been demonstrated by the s-SNOM measurements. The RLC circuit analysis reveals that the nanobridge and the linked rhombic-arm antennas mainly act as the inductance and resistance of the overall structure and determines the resonant frequency and intensity of the high-order mode, as well as those of the fundamental band. The hotspots associated with both bands are spatially superimposed, enabling further boosting up the LFEFs of both bands in a NBRA dimer with a nanogap. Integrating waveguide-cavity coupling, the LFEFs in the nanogap of NBRA dimer-on-reflector structure associated with both bands can be simultaneously improved by up to one order of magnitude in contrast to the NBRA monomer counterpart, thereby achieving monolayer sensitivity for two fingerprints.

We provide a new approach for designing multiband antenna by charge transfer plasmon, efficiently exciting the high-order modes, along with elaborating the importance of the nanobridge and nanogap in MIRAs. These findings also indicate that the island-like metallic films developed as the SEIRA-active substrates in the early stage of SEIRA in 1980s could be considered as nanogap-coupled MIRAs with nanobridges ${ }^{69}$. We believe that designing novel structures with nanobridges accompanied with nanogaps can be a promising strategy for producing multiband MIRAs for general applications in SEIRA, heat-management, and ultrasensitive MIR detectors in the future.

\section{References}

1. Aroca RF, Ross DJ, Domingo C. Surface-enhanced infrared spectroscopy. Appl Spectrosc 58, 324A-338A (2004).

2. Lal S, Grady NK, Kundu J, Levin CS, Lassiter JB et al. Tailoring plasmonic substrates for surface enhanced spectroscopies. Chem Soc Rev 37, 898-911 (2008).

3. Neubrech F, Klevenz M, Meng F, Pucci A. Nanoantennas for surface enhanced infrared spectroscopy. In Cat DT, Pucci A, Wandelt K, eds. Physics and Engineering of New Materials 321-325 (Springer, 2009);

https://doi.org/10.1007/978-3-540-88201-5_37.

4. Neubrech F, Huck C, Weber K, Pucci A, Giessen H. Surfaceenhanced infrared spectroscopy using resonant nanoantennas. Chem Rev 117, 5110-5145 (2017).

5. Yang XX, Sun ZP, Low T, Hu H, Guo XD et al. Nanomaterialbased plasmon-enhanced infrared spectroscopy. Adv Mater 30, 1704896 (2018).
6. Tittl A, John-Herpin A, Leitis A, Arvelo ER, Altug H. Metasurface-based molecular biosensing aided by artificial intelligence. Angew Chem Int Ed 58, 14810-14822 (2019).

7. Taliercio T, Biagioni P. Semiconductor infrared plasmonics. Nanophotonics 8, 949-990 (2019).

8. Caldwell JD, Lindsay L, Giannini V, Vurgaftman I, Reinecke TL et al. Low-loss, infrared and terahertz nanophotonics using surface phonon polaritons. Nanophotonics 4, 44-68 (2015).

9. Wang T, Li PN, Chigrin DN, Giles AJ, Bezares FJ et al. Phonon-polaritonic bowtie nanoantennas: controlling infrared thermal radiation at the nanoscale. ACS Photonics 4, 1753-1760 (2017).

10. Alfaro-Mozaz FJ, Alonso-González P, Vélez S, Dolado I, Autore $\mathrm{M}$ et al. Nanoimaging of resonating hyperbolic polaritons in linear boron nitride antennas. Nat Commun 8, 15624 (2017).

11. Autore M, Li PN, Dolado I, Alfaro-Mozaz FJ, Esteban R et al. Boron nitride nanoresonators for phonon-enhanced molecular vibrational spectroscopy at the strong coupling limit. Light Sci App/ 7, 17172 (2018).

12. Ohtsu M. History, current developments, and future directions of near-field optical science. Opto-Electron Adv 3, 190046 (2020).

13. Novotny L, van Hulst N. Antennas for light. Nat Photonics $\mathbf{5}$, 83-90 (2011).

14. Dong LL, Yang $X$, Zhang $C$, Cerjan B, Zhou LN et al. Nanogapped $\mathrm{Au}$ antennas for ultrasensitive surface-enhanced infrared absorption spectroscopy. Nano Lett 17, 5768-5774 (2017).

15. Chen $\mathrm{C}$, Oh SH, Li M. Coupled-mode theory for plasmonic resonators integrated with silicon waveguides towards mid-infrared spectroscopic sensing. Opt Express 28, 2020-2036 (2020).

16. Chen H, Zhang SY, Miller KA, Braun PV. Autonomic molecular transport for ultrasensitive surface-enhanced infrared absorption spectroscopy. ACS Appl Polym Mater 2, 3929-3935 (2020).

17. Zhang L, Pan J, Zhang Z, Wu H, Yao $\mathrm{N}$ et al. Ultrasensitive skin-like wearable optical sensors based on glass micro/nanofibers. Opto-Electron Adv 3, 190022 (2020).

18. Chen $Q$, Liang $L$, Zheng $Q L$, Zhang $Y X$, Wen L. On-chip readout plasmonic mid-IR gas sensor. Opto-Electron Adv 3 , 190040 (2020).

19. $\mathrm{Yu} \mathrm{N}$, Wang $\mathrm{Q}$, Capasso $\mathrm{F}$. Beam engineering of quantum cascade lasers. Laser Photonics Rev 6, 24-46 (2012).

20. Tong J C, Suo F, Ma J H Z, Tobing L Y M, Qian $L$ et al. Surface plasmon enhanced infrared photodetection. Opto-Electron Adv 2, 180026 (2019).

21. Yao $Y$, Shankar $R$, Rauter $P$, Song $Y$, Kong $J$ et al. High-responsivity mid-infrared graphene detectors with antenna-enhanced photocarrier generation and collection. Nano Lett 14, 3749-3754 (2014).

22. Wang HL, You EM, Panneerselvam R, Ding SY, Tian ZQ. Advances of surface-enhanced Raman and IR spectroscopies: from nano/microstructures to macro-optical design. Light Sci App/ 10, 161 (2021).

23. Novotny L. Effective wavelength scaling for optical antennas. Phys Rev Lett 98, 266802 (2007).

24. Neubrech F, Pucci A, Cornelius TW, Karim S, García-Etxarri A et al. Resonant plasmonic and vibrational coupling in a tailored 
nanoantenna for infrared detection. Phys Rev Lett 101, 157403 (2008).

25. Adato R, Yanik AA, Amsden JJ, Kaplan DL, Omenetto FG et al. Ultra-sensitive vibrational spectroscopy of protein monolayers with plasmonic nanoantenna arrays. Proc Natl Acad Sci USA 106, 19227-19232 (2009).

26. Brown LV, Zhao K, King N, Sobhani H, Nordlander $P$ et al. Surface-enhanced infrared absorption using individual cross antennas tailored to chemical moieties. J Am Chem Soc 135, 3688-3695 (2013).

27. Dregely D, Neubrech F, Duan HG, Vogelgesang R, Giessen H. Vibrational near-field mapping of planar and buried three-dimensional plasmonic nanostructures. Nat Commun 4, 2237 (2013).

28. Huck C, Neubrech F, Vogt J, Toma A, Gerbert D et al. Surfaceenhanced infrared spectroscopy using nanometer-sized gaps. ACS Nano 8, 4908-4914 (2014).

29. Sun $Q, Y u H$, Ueno $K, Z u ~ S$, Matsuo $Y$ et al. Revealing the plasmon coupling in gold nanochains directly from the near field. Opto-Electron Adv 2, 180030 (2019).

30. Neubrech F, Weber D, Lovrincic R, Pucci A, Lopes M et al. Resonances of individual lithographic gold nanowires in the infrared. Appl Phys Lett 93, 163105 (2008).

31. Larkin P. Preface. In Larkin P, ed. Infrared and Raman Spectroscopy: Principles and Spectral Interpretation ix-x (Elsevier, 2011);https://doi.org/10.1016/B978-0-12-386984-5.10013-8.

32. Adato $R$, Yanik AA, Altug $H$. On chip plasmonic monopole nano-antennas and circuits. Nano Lett 11, 5219-5226 (2011).

33. Chen K, Adato R, Altug $\mathrm{H}$. Dual-band perfect absorber for multispectral plasmon-enhanced infrared spectroscopy. ACS Nano 6, 7998-8006 (2012).

34. Turkmen M, Aksu S, Çetin AE, Yanik AA, Altug H. Multi-resonant metamaterials based on UT-shaped nano-aperture antennas. Opt Express 19, 7921-7928 (2011).

35. Cetin AE, Turkmen M, Aksu S, Etezadi D, Altug H. Multi-resonant compact nanoaperture with accessible large nearfields. Appl Phys B 118, 29-38 (2015).

36. Yang $Y$, Dai HT, Sun XW. Fractal diabolo antenna for enhancing and confining the optical magnetic field. AIP Adv 4, 017123 (2014).

37. Gottheim S, Zhang H, Govorov AO, Halas NJ. Fractal nanoparticle plasmonics: the cayley tree. ACS Nano 9, 3284-3292 (2015).

38. Aslan E, Aslan E, Wang R, Hong MK, Erramilli S et al. Multispectral cesaro-type fractal plasmonic nanoantennas. ACS Photonics 3, 2102-2111 (2016).

39. Aouani H, Šípová H, Rahmani M, Navarro-Cia M, Hegnerová K et al. Ultrasensitive broadband probing of molecular vibrational modes with multifrequency optical antennas. ACS Nano $\mathbf{7}$, 669-675 (2013).

40. Bingham CM, Tao H, Liu XL, Averitt RD, Zhang X et al. Planar wallpaper group metamaterials for novel terahertz applications. Opt Express 16, 18565-18575 (2008).

41. Jiang ZH, Yun S, Toor F, Werner DH, Mayer TS. Conformal dual-band near-perfectly absorbing mid-infrared metamaterial coating. ACS Nano 5, 4641-4647 (2011).

42. Rodrigo D, Tittl A, Ait-Bouziad N, John-Herpin A, Limaj O et al. Resolving molecule-specific information in dynamic lipid membrane processes with multi-resonant infrared metasurfaces. Nat Commun 9, 2160 (2018).
43. Olmon RL, Krenz PM, Jones AC, Boreman GD, Raschke MB. Near-field imaging of optical antenna modes in the mid-infrared. Opt Express 16, 20295-20305 (2008).

44. Rang M, Jones AC, Zhou F, Li ZY, Wiley BJ et al. Optical nearfield mapping of plasmonic nanoprisms. Nano Lett 8 , 3357-3363 (2008).

45. Schnell M, García-Etxarri A, Huber AJ, Crozier K, Aizpurua J et al. Controlling the near-field oscillations of loaded plasmonic nanoantennas. Nat Photonics 3, 287-291 (2009).

46. Alonso-González P, Albella P, Schnell M, Chen J, Huth F et al. Resolving the electromagnetic mechanism of surface-enhanced light scattering at single hot spots. Nat Commun 3, 684 (2012).

47. Alonso-González P, Albella P, Golmar F, Arzubiaga L, Casanova $F$ et al. Visualizing the near-field coupling and interference of bonding and anti-bonding modes in infrared dimer nanoantennas. Opt Express 21, 1270-1280 (2013).

48. Olmon RL, Slovick B, Johnson TW, Shelton D, Oh $\mathrm{SH}$ et al. Optical dielectric function of gold. Phys Rev B 86, 235147 (2012).

49. Chen YQ, Xiang Q, Li ZQ, Wang YS, Meng YH et al. "Sketch and peel" lithography for high-resolution multiscale patterning. Nano Lett 16, 3253-3259 (2016).

50. Chen YQ, Bi KX, Wang QJ, Zheng MJ, Liu Q et al. Rapid focused ion beam milling based fabrication of plasmonic nanoparticles and assemblies via "sketch and peel" strategy. ACS Nano 10, 11228-11236 (2016).

51. Chen YQ, Hu YQ, Zhao JY, Deng YS, Wang ZL et al. Topology optimization-based inverse design of plasmonic nanodimer with maximum near-field enhancement. Adv Funct Mater 30, 2000642 (2020).

52. Chen $Y Q$, Shu ZW, Feng ZY, Kong LA, Liu Y et al. Reliable patterning, transfer printing and post-assembly of multiscale adhesion-free metallic structures for nanogap device applications. Adv Funct Mater 30, 2002549 (2020).

53. Chen YQ, Shu ZW, Zhang S, Zeng P, Liang HK et al. Sub-10 $\mathrm{nm}$ fabrication: methods and applications. Int J Extrem Manuf 3, 032002 (2021).

54. Eilers PHC. A perfect smoother. Anal Chem 75, 3631-3636 (2003).

55. Ocelic N, Huber A, Hillenbrand R. Pseudoheterodyne detection for background-free near-field spectroscopy. Appl Phys Lett 89, 101124 (2006).

56. Duan HG, Fernández-Domínguez AI, Bosman M, Maier SA, Yang JKW. Nanoplasmonics: classical down to the nanometer scale. Nano Lett 12, 1683-1689 (2012).

57. Wang T, Dong ZG, Koay EHH, Yang JKW. Surface-enhanced infrared absorption spectroscopy using charge transfer plasmons. ACS Photonics 6, 1272-1278 (2019).

58. Wen FF, Zhang Y, Gottheim S, King NS, Zhang $Y$ et al. Charge transfer plasmons: optical frequency conductances and tunable infrared resonances. ACS Nano 9, 6428-6435 (2015).

59. Zhu D, Bosman M, Yang JKW. A circuit model for plasmonic resonators. Opt Express 22, 9809-9819 (2014).

60. Huang $\mathrm{CP}, \mathrm{Yin} X \mathrm{XG}$, Huang $\mathrm{H}$, Zhu $\mathrm{YY}$. Study of plasmon resonance in a gold nanorod with an LC circuit model. Opt Express 17, 6407-6413 (2009).

61. Khurgin JB, Sun G. Scaling of losses with size and wavelength in nanoplasmonics and metamaterials. Appl Phys Lett 99, 
211106 (2011).

62. Zhou J, Koschny T, Kafesaki M, Economou EN, Pendry JB et al. Saturation of the magnetic response of split-ring resonators at optical frequencies. Phys Rev Lett 95, 223902 (2005).

63. Giannini V, Francescato $\mathrm{Y}$, Amrania H, Phillips CC, Maier SA. Fano resonances in nanoscale plasmonic systems: a parameter-free modeling approach. Nano Lett 11, 2835-2840 (2011).

64. Neuman T, Alonso-González P, Garcia-Etxarri A, Schnell M, Hillenbrand $R$ et al. Mapping the near fields of plasmonic nanoantennas by scattering-type scanning near-field optical microscopy. Laser Photonics Rev 9, 637-649 (2015).

65. Ahmed A, Gordon R. Single molecule directivity enhanced raman scattering using nanoantennas. Nano Lett 12, 2625-2630 (2012).

66. Wang DX, Zhu WQ, Best MD, Camden JP, Crozier KB. Directional raman scattering from single molecules in the feed gaps of optical antennas. Nano Lett 13, 2194-2198 (2013).

67. Brown LV, Yang X, Zhao K, Zheng BY, Nordlander $\mathrm{P}$ et al. Fan-shaped gold nanoantennas above reflective substrates for surface-enhanced infrared absorption (SEIRA). Nano Lett 15, 1272-1280 (2015).

68. Born MAX, Wolf E. Basic properties of the electromagnetic field. In Born MAX, Wolf E, eds. Principles of Optics: Electromagnetic Theory of Propagation, Interference and Diffraction of Light 1-70 (Pergamon, 1980); https://doi.org/10.1016/B978-0-08-026482-0.50008-6.

69. Masatoshi O. Dynamic processes in electrochemical reactions studied by surface-enhanced infrared absorption spectroscopy
(SEIRAS). Bull Chem Soc Jpn 70, 2861-2880 (1997).

\section{Acknowledgements}

This work was supported by the National Natural Science Foundation of China (Grant No. 21403179, 21727807, 51722503, 51805160) and the Science and Technology Bureau Foundation of Changsha City (kh1904005). We acknowledge the support from the State Key Laboratory of Physical Chemistry of Solid Surfaces, Xiamen University, for providing the s-SNOM system. We cordially acknowledge Sergiu Amarie, Felix Blanck and Claas Reckmeier from neaspec for helpful discussion on the s-SNOM imaging, and thank Dana Cialla-May from Friedrich Schiller University Jena for the sample fabrication in the early stage.

\section{Author contributions}

S. Y. Ding conceived the idea. E. M. You and J. Yi performed the simulations. Y. Q. Chen and H. G. Duan fabricated the structures and measured the SEM of the NBRA structure. E. M. You performed the micro-FTIR and s-SNOM measurements. E. M. You and Z. D. Meng performed the RLC circuit modelling. Q. Chen and E. M. You measured the SEM of the cross section of the reflective substrate. S. Y. Ding and H. G. Duan supervised the project. E. M. You, Y. Q. Chen, J. Yi, S. Y. Ding, H. G. Duan, M. Moskovits and Z. Q. Tian contributed to the writing of the manuscript.

\section{Competing interests}

The authors declare no competing financial interests.

\section{Supplementary information}

Supplementary information for this paper is available at https://doi.org/10.29026/oea.2021.210076 